\title{
AIRCRAFT OPERATION DEPENDING UPON THE UNCERTAINTY OF MAINTENANCE ALTERNATIVES
}

\author{
Andriy GONCHARENKO \\ National Aviation University, 1, Kosmonavta Komarova Ave., Kyiv, 03680, Ukraine \\ E-mail: andygoncharenco@yahoo.com
}

Received 13 April 2017; accepted 27 November 2017

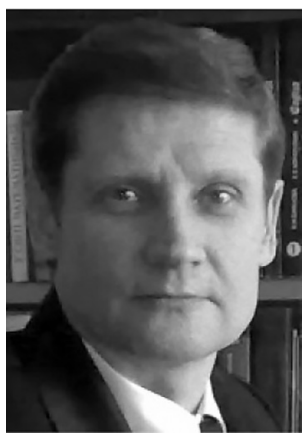

Andriy GONCHARENKO, Assoc Prof, Dr Sci (Eng)

Date of birth: 1962.

Education: Odesa Institute of Marine Engineers, 1984.

Affiliations and functions: professor in the Department of Aircraft Airworthiness, at the National Aviation University, Kyiv, Ukraine.

Research interests: safety of active systems, variational principles of operation of power plants in multi-alternative situations, control theory, flight dynamics, data processing, identification, organizational systems.

Publications: author of over 90 scientific papers.

Abstract. The paper considers the theoretical explanation and construction of some mathematical models of the aircraft operational process in reference to maintenance organizations preferred by aviation experts and aircraft operators. The uncertainty of such operational multi-alternativeness is evaluated on the basis of subjective entropy of preferences demonstrated by aircraft operators and aviation experts. When applying the maximum principle of subjective entropy, the optimal distributions of the preferences are obtained. The proposed concept allows finding the optimal distribution of the aircraft fleet according to the available maintenance alternatives. To optimize the above mentioned distribution, one may take into account the possible development and improvement of maintenance organizations and so called "shadow" components. The paper also discusses a significant approach that helps to evaluate the effectiveness of organizations functioning in the area of aviation. This evaluation can be carried out at both global and continental levels as well as state, operator, and airline levels. The respective modeling has been performed and is illustrated with diagrams.

Keywords: aircraft operation, airworthiness support, flight safety, aircraft maintenance, multi-alternativeness, uncertainty, maximum principle of subjective entropy, optimization, shadow economy, individual preferences.

\section{Introduction}

Aircraft operation is carried out under uncertainty regarding operational conditions. This uncertainty leads to variations in the timetables of flight departures and arrivals. It should be noted that unpredictable events which cause incidents or accidents may occur sporadically. Besides, such episodes take place due to both failures in aeronautical engineering, as considered in (Dhillon 2006) and (Smith 2005), and human factors. Under the prism of this study, there is a lack of understanding that maintainability, reliability, and risk, in the comprehension presented by (Dhillon 2006) and (Smith 2005) mentioned above, deal with probabilities as an objective characteristic of an engineering system in the broadest sense, thus, losing from the sight the importance of individuals' subjective characteristics which are the preferences of alternatives.

Now, considering the topical problems of the aviation industry in more detail, it should be noted that this fact ought to be taken into account when carrying out aircraft maintenance (Dmitriev et al. 2015). Considering the possible ways, methods, and techniques for maintenance process improvement, a responsible person driven by his/her own personal, individual, and subjective, scale of the preferences distribution system makes the final decision concerning, in his/her opinion, some better options, keeping in mind such indispensable issues as fight safety and airworthiness levels but not only those. 
Flight safety precautions are required by the ICAO, EASA, and National Civil Aviation Authorities (NCAA) in the field of aircraft operation and its airworthiness support. These requirements include scheduled maintenance or periodical maintenance according to aircraft conditions at officially approved maintenance organizations.

The aircraft maintenance organizations (AMOs) compete in order to receive the permission to maintain and overhaul airlines' aircraft fleets (AAFs), as well as to achieve the business enhancement and promotion in the global market owing to the officially approved certification. Therefore, AMOs enlarge their spheres of interests and influences, offering either improved services or advanced technologies. From time to time, their own customers, i.e. airlines (AAFs/aircraft operators), are given some other privileges.

Thus, aircraft operators (AOs) face specific operational situations related to a multiple choice for a proper AMO under conditions of uncertainty deriving from multi-alternativeness for the AAFs airworthiness support and flight safety measures.

Consequently, the AOs use the assessment performed by aviation experts (similarly to ICAO, EASA, or NCAA inspections and procedures for AMOs approval) or another kind of estimation, for instance, examinations in order to make better or even the best decisions in some respect (Yuttapong, Sataporn 2012). This paper considers the applicability of models for providing an appraisal for Thailand based aircraft maintenance with regard to multi-objective optimization, which is a bit closer to the multi-alternativeness of operational situations. However, a study gap, requiring endeavors to undertake scientifically substantiated research has been identified, since the paper's decision-support model does not use any functions of alternatives subjective preferences, derived on the basis of some variational or optimization principle in combination with an explainable uncertainty degree measure, in an explicit way.

The background of this topic in conjunction with past studies, and the identified corresponding literature source deficiencies, encourage urgent attempts for developing an adequate theoretical basis encompassing some simplest, to a marginal extent, demonstrative models allowing to investigate the principal dependences between the functions variables related to the participants in aircraft airworthiness support processes.

The objective of the research presented here is to solve the problem described above in the framework of subjective analysis developed by V. Kasianov, professor of the National Aviation University, Kyiv, Ukraine, in order to determine certain optimal values indicating the influence of aircraft operators on the characteristics of AAFs functioning with respect to aircraft airworthiness and maintenance (Kasianov 2013; Kasianov, Goncharenko
2013; Goncharenko 2016). Subjective analysis (the theory of subjective preferences) is an appropriate tool using the subjective preferences entropy paradigm embodied in the view of the subjective entropy maximum principle (SEMP) for solving the problems of optimal distributions of individuals' subjective preferences taking into account the situational uncertainty of aircraft operational alternatives. The SEMP postulated in subjective analysis (Kasianov 2013) allows taking into consideration optimally distributed subjective preferences functions, derived mathematically from an objective functional, explicitlly in order to assess the responsible persons' intentions.

Subjective analysis, in turn, requires further development and needs more application fields and extended testing of the applicability of the mathematical models at least in view of numerical simulations.

Our research deals with several important aspects of subjective quality evaluation in aviation maintenance, also considered in (Gališanskis 2004), similarly for the assessment, prediction, and control of aviation noise see (Zaporozhets et al. 2011).

These are also, to a certain degree, adjacent scientific problems that are related to the cornerstone of the subjective preferences theory - the SEMP or at least some dealing with its neighboring areas.

\section{Schematic consideration of the problem statement}

To formulate the research problem for the analysis of aircraft operation processes of maintenance and airworthiness support taking into account the subjective preferences of the process participants towards maintenance alternatives, it is recommended to schematically consider the aircraft operation process shown in Figure 1, which depends on alternative AMOs.

In Figure 1, the three layers are depicted as follows: $\mathrm{AO}$ - AO; $\operatorname{Exp}_{1}, \mathrm{Exp}_{2}$ - two experts; $\mathrm{AMO}_{\mathrm{I}}, \mathrm{AMO}_{\mathrm{II}}$ - two AMOs. Also, there are two flows of AAF maintenance - I and II. The arrows symbolize certain resources (information, preferences, "shadow" or "light" financing, aircraft for maintenance, etc.) and the directions of their flow.

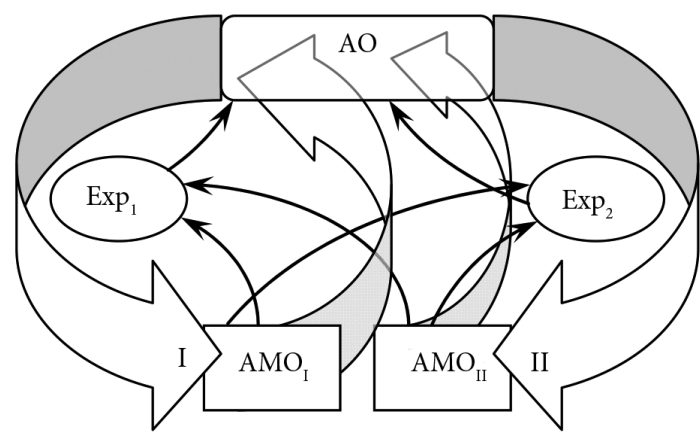

Fig. 1. Information-resource flow-chart diagram of aircraft fleet distribution between two maintenance organizations 
The flow-chart diagram in Figure 1 illustrates the possibilities of an AAF maintenance process. The role of the experts shown in Figure 1 is a very specific one, and thus important, because they are deemed to be independent assessors of the two AMOs, being at the same time independent advisors of the AO with regard to splitting the AAF for maintenance. Also, considering the process shown in Figure 1, the important fact is that the experts' intentions are driven by some kind of a "divisor" between "light" and "shadow" components of their business. The monograph by Kasianov, Goncharenko (2013) is dedicated to the "shadow" economy proportion levers; this approach is applied as patterns for both the problem statement and the solution.

In the simplest case, it is assumed that the AO makes its own decision concerning the distribution of the AAF between the two flows in accordance with its own subjective preferences pertaining to both AMOs. The AO's preferences are formed under the impact (influence) of the AMOs' effectiveness functions themselves as well as the experts' subjective preferences for the AMOs with respect to the AO's opinion.

\section{General methods of research}

The problem statement is slightly similar but not absolutely analogous to a Markovian random process; the intrinsic matter appears to be the preferences for the quantitative characteristics of AAFs' maintenance flows, but not the probabilities of transitions for a random process with discrete states and continuous time. Although, the possible relations between the preferences and probabilities connected with the transition rates and intensities are not denied.

\subsection{Experts' preferences regarding alternative aircraft maintenance organizations}

It is assumed that the experts' (inspectors') preferences are formed under the influence of the two alternative AMOs effectiveness functions expressed as some quantitative score values independent upon each other. In the system of aircraft fleet distribution (see Fig. 1), no one knows the other subject's ratings; each knows only what is received or given.

These initial simplifications allow finding the experts' preferences with the help of the SEMP, proposed in (Kasianov 2013) and successfully applied to a variety of problems described in (Kasianov 2013; Kasianov, Goncharenko 2013; Goncharenko 2016). Such simplifications imply the uncertainty of aircraft operational multialternativeness in view of the preferences distribution entropy related to the availability of AMOs.

In (Kasianov, Goncharenko 2013), it is emphasized that the preferences are generated on the basis of a certain variational principle. One can surmise that the extremized operational purpose functional has the following view:

$$
\begin{aligned}
& \Phi_{\pi_{1}}=-\pi_{1}^{I} \ln \pi_{1}^{I}-\pi_{1}^{I I} \ln \pi_{1}^{I I}-\beta \pi_{1}^{I} \ln C_{1}^{I I}- \\
& \beta \pi_{1}^{I I} \ln C_{1}^{I}+\gamma\left(\pi_{1}^{I}+\pi_{1}^{I I}-1\right),
\end{aligned}
$$

where $\Phi_{\pi_{1}}$ is the operational functional value extremized by the first expert on the set of the two achievable alternatives of available AMOs; $\pi_{1}^{I}$ - the preference of the first AMO by the first inspector; $\pi_{1}^{I I}$ - the preference of the first inspector for the second AMO; $\beta$ - an endogenous parameter of the inspector's attitude to the alternatives; $C_{1}^{I I}$ - the special utility/effectiveness/cognitive dimensionless function (possibly ratio) which allows to conduct mathematically logarithmic operations. This function is a score or even might be a contribution to the subjective attractiveness of the alternatives or a shadow financial component. This attractiveness, in turn, has been obtained by the second AMO in the first expert's opinion; $C_{1}^{I}$ is thus the corresponding cognitive function of the first inspector for the first AMO and $\gamma$ is the Lagrange multiplier.

$$
-\pi_{1}^{I} \ln \pi_{1}^{I}-\pi_{1}^{I I} \ln \pi_{1}^{I I} .
$$

Expression (2) shows a measure of uncertainty (entropy) of the first inspector's preferences with regards to $\mathrm{AMO}_{\mathrm{I}}$, and $\mathrm{AMO}_{\mathrm{II}}$, as seen in Figure 1 (subscript 1 and superscripts $I, I I$ by the designation of preference $\pi$ ).

$$
-\beta \pi_{1}^{I} \ln C_{1}^{I I}-\beta \pi_{1}^{I I} \ln C_{1}^{I} .
$$

Expression (3) is the cognitive function, effectiveness function, and the member where $\beta>0$, because the ideology of the operational objective functional of Equation (1) implies an increase in a certain AMO's preference in case of the competing AMO's significance decreases, i.e. the first inspector prefers the first AMO more, if he/she gains less benefit from the second AMO (both AMOs' benefits are in the logarithmic scale). The same pertains to the second AMO.

$$
\left(\pi_{1}^{I}+\pi_{1}^{I I}-1\right)
$$

Expression (4) denotes the normalizing condition for the first inspector's preferences for the operational alternatives of $\mathrm{AMO}_{\mathrm{I}}$, and $\mathrm{AMO}_{\mathrm{II}}$ (see Fig. 1).

In accordance with the SEMP, the distributions of the so-called canonical view preferences are obtained with the use of the necessary conditions for the extremum existence in Equation (1):

$$
\frac{\partial \Phi_{\pi_{1}}}{\partial \pi_{1}^{I}}=-\ln \pi_{1}^{I}-1-\beta \ln C_{1}^{I I}+\gamma=0 .
$$

The application of the same condition for the preference of the second AMO by the first expert, as in Equation (5), and use of the normalizing condition in 
Equation (4) yield the desirable extremal canonical view distribution for the following preferences:

$$
\pi_{1}^{I}=\frac{\left(C_{1}^{I I}\right)^{-\beta}}{\left(C_{1}^{I}\right)^{-\beta}+\left(C_{1}^{I I}\right)^{-\beta}} ; \quad \pi_{1}^{I I}=\frac{\left(C_{1}^{I}\right)^{-\beta}}{\left(C_{1}^{I}\right)^{-\beta}+\left(C_{1}^{I I}\right)^{-\beta}} .
$$

The method described in Equations (1-6) is also applied to obtain the second expert's preferences. Besides, the endogenous parameter of the inspectors' attitude to the alternatives, $\beta$, is considered to be identical for both of them, which is one more simplification assumed at the problem statement.

The model of Equations (1-6) does not contain the extremal values for the preferences regarding the two independent variables of the shadow components: $C_{1}^{I}$ and $C_{1}^{I I}$.

$$
\frac{\partial \pi_{1}^{I}}{\partial C_{1}^{I I}}=\frac{-\beta\left(C_{1}^{I I}\right)^{-\beta-1}\left(C_{1}^{I}\right)^{-\beta}}{\left[\left(C_{1}^{I}\right)^{-\beta}+\left(C_{1}^{I I}\right)^{-\beta}\right]^{2}}=0
$$

If the conditions of Equation 7 are fulfilled, it leads to a contradiction, since neither $\beta$ nor $C_{1}^{I I}$, nor $C_{1}^{I}$ equal zero. Even if the condition of Equation 7 had been satisfied, it would not have given much since the second derivative of Equation (6), i.e. the first derivative of Equation (7), would have equalled zero, which would have meant additional research.

The other model involves modifying the operational purpose functional Equation (1) and using the SEMP:

$$
\begin{aligned}
& \Phi_{\pi_{1}}=-\pi_{1}^{I} \ln \pi_{1}^{I}-\pi_{1}^{I I} \ln \pi_{1}^{I I}+\beta \pi_{1}^{I} \ln C_{1}^{I}+ \\
& \beta \pi_{1}^{I I} \ln C_{1}^{I I}+\gamma\left(\pi_{1}^{I}+\pi_{1}^{I I}-1\right) .
\end{aligned}
$$

Here in Equation (8), contrary to Equation (1) and Expression (3), the more benefit the first inspector gains from the first AMO, the more he/she prefers it (analogically to Equation (1) and Expression (3) in the logarithmic scale). The same pertains to the second AMO. In the cognitive and effectiveness functions of Equation (8), as well as in Equation (1) and Expression (3), $\beta>0$.

Optionally, it is appropriate to conduct the modelling with exponential 'divisors' for different participants with different combinations (Kasianov, Goncharenko 2013).

The subjective entropy approach shown in Equations (1-8) provides the possibility for modelling the AMOs' influence via their experts' assessments on the AOs choices regarding their aircraft fleet maintenance.

The levels of the expert assessment uncertainties concerning the certain AMOs' abilities to carry out the AOs' AAFs maintenance are modelled with the experts' individual preferences subjective entropies described in Expression (2).

\subsection{Aircraft operators' preferences taking into account the experts' preferences}

To get the models of the AO's preferences, the exponential "divisor" in the framework of the SEMP is used (Kasianov, Goncharenko 2013):

$$
\begin{aligned}
& \Phi_{\pi_{A O}}=-\pi_{I} \ln \pi_{I}-\pi_{I I} \ln \pi_{I I}-\beta \pi_{I}\left(F_{I} \pi_{1}^{I} \pi_{2}^{I}\right)- \\
& \beta \pi_{I I}\left(F_{I I} \pi_{1}^{I I} \pi_{2}^{I I}\right)+\gamma\left(\pi_{I}+\pi_{I I}-1\right),
\end{aligned}
$$

where $\Phi_{\pi_{A O}}$ is the operational purpose functional value optimized (extremized) by the $\mathrm{AO}$ on the set of the two achievable alternatives of the possible AMOs; $\pi_{I}$ - the preference of the first AMO; $\pi_{I I}$ - the preference of the second AMO; $\beta$ - the endogenous parameter of the AO's attitude to the alternatives (it is deemed to be the same by its absolute value $|\beta|$ as of the inspectors' value, which also simplifies the problem statement as an assumption, although in Equation (9), with the same module $|\beta|$, it is a negative value, i.e. $\beta<0) ; F_{I}$ and $F_{I I}$ - cognitive functions (utility/score) obtained by the first and second AMO in the AO's opinion respectively; $\pi_{2}^{I}$ and $\pi_{2}^{I I}$ - the corresponding preferences of the second inspector for $\mathrm{AMO}_{\mathrm{I}}$, and $\mathrm{AMO}_{\mathrm{II}}$ (see Fig. 1).

For the AO's operational purpose functional value optimization model in view of Equation (9), an assumption of his/her complete evaluation of the experts' preferences trustworthiness in relation with the two AMOs has been accepted. Although, some weight coefficients might be used in order to take into account the ratings and reliability of the inspectors and their preferences' valuable potential for the AO. Also, it is possible to model the relations between $F_{I}$ and $F_{I I}$, as well as with $\pi_{1}^{I}\left(C_{1}^{I}, C_{1}^{I I}\right)$, $\pi_{1}^{I I}\left(C_{1}^{I}, C_{1}^{I I}\right), \pi_{2}^{I}\left(C_{2}^{I}, C_{2}^{I I}\right), \pi_{2}^{I I}\left(C_{2}^{I}, C_{2}^{I I}\right), C_{1}^{I}, C_{1}^{I I}, C_{2}^{I}$, and $C_{2}^{I I}$; here $C_{2}^{I}$ and $C_{2}^{I I}$ are the corresponding contributions of $\mathrm{AMO}_{\mathrm{I}}$ and $\mathrm{AMO}_{\mathrm{II}}$ to the cognitive functions of the second inspector.

The necessary conditions of the Equation (9) extremum and analogous approaches such as Equation (5) to the $\mathrm{AMO}_{\mathrm{I}}$ yield as follows:

$$
\frac{\partial \Phi_{\pi_{A O}}}{\partial \pi_{I}}=-\ln \pi_{I}-1-\beta\left(F_{I} \pi_{1}^{I} \pi_{2}^{I}\right)+\gamma=0 .
$$

From here, by applying a similar approach to Equation (10) for the AO's preference of the $\mathrm{AMO}_{\mathrm{II}}$, we obtain the AO's extremal preferences distribution in the canonical view similar to Equation (6) in the exponential form via:

$$
\begin{aligned}
& \pi_{I}=\frac{\exp \left[-\beta\left(F_{I} \pi_{1}^{I} \pi_{2}^{I}\right)\right]}{\exp \left[-\beta\left(F_{I} \pi_{1}^{I} \pi_{2}^{I}\right)\right]+\exp \left[-\beta\left(F_{I I} \pi_{1}^{I I} \pi_{2}^{I I}\right)\right]} ; \\
& \pi_{I I}=\frac{\exp \left[-\beta\left(F_{I I} \pi_{1}^{I I} \pi_{2}^{I I}\right)\right]}{\exp \left[-\beta\left(F_{I} \pi_{1}^{I} \pi_{2}^{I}\right)\right]+\exp \left[-\beta\left(F_{I I} \pi_{1}^{I I} \pi_{2}^{I I}\right)\right]} .
\end{aligned}
$$




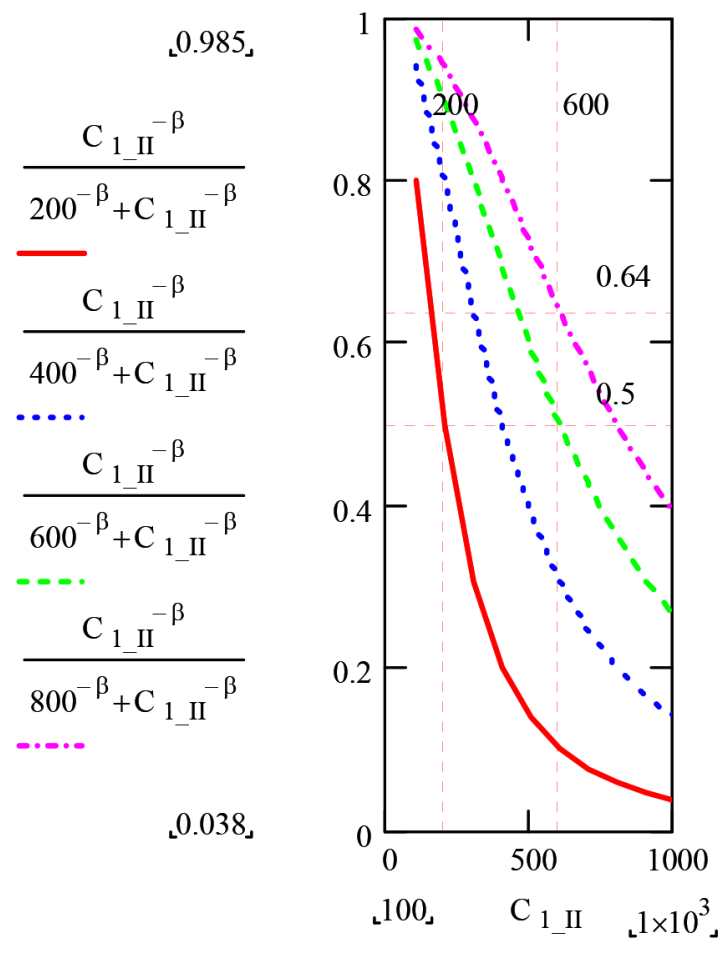

Fig. 2. Changes of the first expert's preferences for the first maintenance organization

Thus, the AO's preferences distribution is received for the two specified AMOs, taking into consideration the uncertainty at both the AO's and experts' related to the AMOs' levels. The AO's preferences for the two above mentioned AMOs are the functions of four independent variables: $C_{1}^{I}, C_{1}^{I I}, C_{2}^{I}$, and $C_{2}^{I I}$, and the AMOs' utility for $\mathrm{AO}$, i.e. $F_{I}$ and $F_{I I}$ cognitive functions, are constant values that have been considered for the described problem statement so far for ease of explanation.

It is natural to believe that the $\mathrm{AO}$ is going to divide the aircraft fleet to dispatch it for maintenance at those AMOs in the proportion of the preferences distribution ratios. Also, apparently, the final AO's decisions concerning the appropriate AAFs AMOs are made at some levels of uncertainty measured with the AO's subjective entropies as in Expression (2).

Using their own resources, the AMOs can influence the AO's choice and displace the balance of the flow of aircraft for maintenance and overhaul in their favour by means of experts' preferences, with the use of $C_{1}^{I}, C_{1}^{I I}$, $C_{2}^{I}$, and $C_{2}^{I I}$, as well as their own attractiveness described as $F_{I}$ and $F_{I I}$ from the AO's point of view (see Fig. 1).

\section{Results and discussion}

As a result, so far we have obtained the canonical distributions of the preferences for AMOs, Equation (6) and Equation (11), which provide the possibility to trace the changes in the preferences.

For example, for Equation (6), these changes are shown in Figure 2. The required calculation testing has been conducted with the use of a standard MathCad computing platform.

In Figure 2, $\mathrm{C}_{1 \_\mathrm{II}}$ stands for $C_{1}^{I I}$, whereas $C_{1}^{I}$ takes the values of $200,400,600$, and 800 , respectively.

The numerical data required for plotting the diagrams for the calculation experiments (see Fig. 2) has been accepted to be as follows: $\left.\left.C_{1 \_ \text {II }}=\right] 0 \ldots 1 \cdot 10^{3}\right]$; $\beta=2$.

The units of the parameters used in the numerical simulation are conditional, relative units can also be used. This does not diminish the significance of the results, because, due to the proposed methodology, one is capable of assessing the subjective preferences influencing the aircraft maintenance and airworthiness support processes.

For the example presented here (Fig. 2), the value of the second AMO's shadow influence exerted upon the first inspector is at the level of $C_{1}^{I I}=200$. Then, if the influence of the competing AMO, the first one, is also 200 conditional relative units, the preference of the first AMO by the first expert (obtained with the help of the first formula of (6)) will be at the level of 0.5. This case is shown in Figure 2 where the red solid curve is crossed by the corresponding coordinates' lines - $(200 ; 0.5)$. The other case, where $C_{1}^{I I}=600$ and $C_{1}^{I}=800$, is depicted in Figure 2 as well. The first expert's preference for the first AMO, consequently, reaches the level of 0.64 , visible when the lily-burgundy dash-dot trace is crossed by the corresponding coordinates' lines of $(600 ; 0.64)$.

It has to be emphasised that the matter investigated in this paper represents just one of the possible concepts for the "expert"/"maintenance organization"/"airline" relations illustrated in Figures 1 and 2 and mathematically described with the help of functionals (1), (8) and (9) on the basis of the SEMP.

Concerning the values of the models expressed with the parameters of Equations (1)-(11), the considered problem is also disputable, especially with regard to the endogenous parameter $\beta$.

Nevertheless, the situation when both AMOs have shadow influences on the expert is similar: for the first expert we have $C_{1}^{I}=C_{1}^{I I}$ and his/her preferences for the AMOs are also the same and equal 0.5, which seems quite logical and pretty truthful.

As to the inequality variant: $C_{1}^{I} \neq C_{1}^{I I}$, the preference function is inclined towards the side of a more attractive operational alternative but not in a purely linearproportion style, which we assume is fair enough.

\section{Conclusions}

The optimal distribution process of AAFs for the available AMOs can be modelled with respect to the optimal values of the process participants. Each time we evaluate the uncertainty of the optional multi-alternativeness, 
the corresponding preferences entropy (Expression (2)) is used.

Further research based on the proposed approach has some perspectives for the investigation of conditional optimization and computer simulation in order to illustrate the theoretical speculations expressed with Equations (1)-(11).

\section{References}

Dhillon, B. S. 2006. Maintainability, maintenance, and reliability for engineers. New York: Taylor \& Francis Group. 214 p. https://doi.org/10.1201/9781420006780

Dmitriev, S.; Burlakov, V.; Popov, O.; Popov, D. 2015. Technological processes and quality control in aircraft engine maintenance, Aviation 19(3): 133-137. https://doi.org/10.3846/16487788.2015.1107306

Gališanskis, A. 2004. Aspects of quality evaluation in aviation maintenance, Aviation 8(3): 18-26.

Goncharenko, A. 2016. Several models of artificial intelligence elements for aircraft control, in IEEE 4th International Con- ference "Methods and Systems of Navigation and Motion Control (MSNMC)", 18-20 October 2016, Kyiv, Ukraine, 224-227.

Kasianov, V. 2013. Subjective entropy of preferences. Subjective analysis: monograph. Warsaw, Poland: Institute of aviation. $644 \mathrm{p}$.

Kasianov, V.; Goncharenko, A. 2013. Light and shadow. Proportions of shadow economy. Entropy approach: monograph. Kyiv, Ukraine: "Kafedra". 86 p.

Smith, D. J. 2005. Reliability, maintainability and risk. Practical methods for engineers. London: Elsevier. 365 p.

Yuttapong, P.; Sataporn, A. 2012. Multiobjective optimization of aircraft maintenance in Thailand using goal programming: a decision-support model, Advances in Decision Sciences 2012, Article ID 128346. 17 p. [online], [cited 16 March 2016]. Available from Internet: http://www.hindawi. com/journals/ads/2012/128346/

Zaporozhets, O.; Tokarev, V.; Attenborough, K. 2011. Aircraft noise. Assessment, prediction and control. Glyph International, Tailor and Francis. $480 \mathrm{p}$. https://doi.org/10.1201/b12545 